\title{
Hypomagnesaemia and its determinants in a contemporary primary care cohort of persons with type 2 diabetes
}

\author{
Femke Waanders ${ }^{1} \cdot$ Robin P. F. Dullaart ${ }^{2} \cdot$ Michel J. Vos ${ }^{3}$ Steven H. Hendriks ${ }^{4} \cdot$ Harry van Goor ${ }^{5} \cdot$ Henk J. G. Bilo $^{6}$. \\ Peter R. van Dijk $\mathbb{D}^{2,7}$
}

Received: 1 August 2019 / Accepted: 13 October 2019 / Published online: 24 October 2019

(C) The Author(s) 2019

\begin{abstract}
Aims Among persons with type 2 diabetes mellitus (T2DM) hypomagnesaemia has been reported in 14-48\% of patients. This may be of significance given the emerging associations of hypomagnesaemia with glucometabolic disturbances and possibly even complications. We assessed the prevalence of hypomagnesaemia and its determinants, in a well-defined cohort of persons with T2DM treated in primary care.

Methods Observational cohort study among persons with T2DM treated in primary care in the Northeast of the Netherlands. Magnesium was measured using a colorimetric endpoint assay (Roche). Hypomagnesaemia was defined as a serum magnesium level $<0.70 \mathrm{mmol} / \mathrm{L}$. Pearson correlations were performed to correlate variables with serum magnesium. Next, a stepwise backward regression model was made.

Results Data of 929 persons (55\% male) with a mean age of $65( \pm 10)$ years, diabetes duration 6.5 [3.0-10.1] years, and HbAlc concentration $6.7( \pm 0.7) \%(50( \pm 9) \mathrm{mmol} / \mathrm{mol})$ were analysed. Serum magnesium was $0.79( \pm 0.08) \mathrm{mmol} / \mathrm{L}$. The percentage of persons with magnesium deficiency was $9.6 \%$. Age, diabetes duration, BMI, HbA1c, use of metformin, sulfonylurea derivatives, and DPP4 inhibitors were negatively associated with magnesium concentrations. In contrast, LDL cholesterol and serum creatinine were positively associated serum magnesium.

Conclusions Hypomagnesaemia was present in $9.6 \%$ of T2DM patients treated in primary care. This percentage is remarkably lower than reported previously, possibly due to the unselected nature of our population. Concerning T2DMrelated factors, only BMI, HbA1c and the use of metformin, sulfonylurea derivatives and DPP4 inhibitors correlated negatively with magnesium concentrations.
\end{abstract}

Keywords Type 2 diabetes $\cdot$ Magnesium $\cdot$ Hypomagnesaemia $\cdot$ Prevalence

Peter R. van Dijk

p.r.van.dijk@umcg.nl

1 Department of Nephrology, Isala, Zwolle, The Netherlands

2 Department of Endocrinology, University Medical Center, University of Groningen, Groningen, The Netherlands

3 Department of Clinical Chemistry, Isala, Zwolle, The Netherlands

4 Department of General practice, University of Leiden, Leiden, The Netherlands

5 Department of Pathology and Medical Biology, University Medical Center, University of Groningen, Groningen, The Netherlands

6 Department of Internal Medicine, University Medical Center, University of Groningen, Groningen, The Netherlands

7 Diabetes Centre, Isala, Zwolle, The Netherlands

\section{Introduction}

Over the past decades, the relevance of abnormalities in magnesium metabolism among persons with type 2 diabetes mellitus (T2DM) is increasingly appreciated [1]. Low concentrations of magnesium in persons with T2DM have been attributed to (poor) dietary intake, autonomic dysfunction, altered insulin metabolism, glomerular hyperfiltration, osmotic diuresis, metabolic acidosis, hypophosphataemia, and hypokalaemia [2]. Low magnesium concentrations are also linked to poor glycaemic control and the presence of micro- and macrovascular complications. Moreover, plasma magnesium was found to predict the risk of incident T2DM in a large cohort from the north of the Netherlands [3]. Of note, supplementation of magnesium is easy, low-cost and was shown to improve 
insulin sensitivity [4], glucose metabolism [5, 6] and to retard progression from pre-diabetes to overt diabetes [7].

Hypomagnesaemia in persons with T2DM has been reported to vary between 14 and $48 \%$ [2]. However, most of these studies were performed within selected populations [8], under clinical circumstances [9, 10], included both adults and children [10], included a relatively low number of participants $[9,11,12]$ or included participants treated in a secondary or tertiary care setting [13]. In addition, some of these studies were performed over 20 years ago and did not take the influence of novel treatment options for DM and associated conditions (i.e. DPP4 inhibitors or statins) into account.

Given the increasing incidence of DM, the potential importance of disturbances in magnesium metabolism and potential for supplementation we aimed to investigate the prevalence of hypomagnesaemia in a well-characterised, but unselected cohort of outpatients with T2DM treated in primary care. In addition, determinants associated with serum magnesium concentrations were identified.

\section{Patients and methods}

Clinical data and blood samples were obtained from the prospective, observational cohort e-VitaDM study, which was designed to assess the feasibility of using an online platform in routine primary healthcare for subjects with T2DM. As a pre-specified part of the e-VitaDM study, participants were assessed in a long-term follow-up protocol. This prospective arm was nested within the Zwolle Outpatient Diabetes project Integrating Available Care (ZODIAC) study. Both the e-VitaDM and the ZODIAC study are described in detail elsewhere [14]. Primary aim of the present study was to investigate the prevalence of hypomagnesaemia in T2DM outpatients. As secondary outcomes the cross-sectional associations between baseline concentrations of magnesium with indices of T2DM management, including $\mathrm{HbA} 1 \mathrm{c}$ and the presence of complications, were assessed.

This study was conducted in general practices that are connected to the Care Group Drenthe in the Drenthe-region (located in the North-East) of the Netherlands: 42 out of the 110 general practices in this care group agreed to participate. In these practices, $~ 8300$ persons were treated for their T2DM between 2012 and 2014. Participants were recruited during a regular check-up by their practice nurse and included from May 2012 until September 2014 [14, 15]. Persons with T2DM, aged $\geq 18$ years and the general practitioner as main care provider for T2DM were eligible for participation [14]. Only patients who were co-treated in secondary care (for their DM), who were terminally ill or had dementia were excluded. A total of 1710 out of 3988 participants, who were asked to participate in the eVita-DM study, gave written informed consent. Of these persons, 764 were not included in the present study due to the inability to match blood samples taken in the e-VitaDM with the clinical data from the ZODIAC study. A further 17 individuals were excluded from the final analysis because of insufficient blood material to perform the measurements. Consequently, the final study cohort consisted of 929 participants.

Baseline demographic data included age, gender, diabetes duration, body mass index (BMI), alcohol intake and smoking habits. Information concerning alcohol intake and smoking habits was derived from questionnaires at baseline. Medical data were extracted from the diabetes-specific database at the Diabetes Centre of the Isala hospital (Zwolle, The Netherlands). This centre gathers data of primary care-treated persons with T2DM in a large part of the Netherlands on an annual basis, to provide benchmark information to general practitioners. This database includes information on physical examination, use of medication, and laboratory blood and urine tests. The following data were extracted: date of diabetes diagnosis, height, weight, diastolic and systolic blood pressure, cholesterol, HbA1c, estimated glomerular filtration rate based on the modification of diet in renal disease (MDRD) equation, urine creatinine, urine albumin, urine albumin:creatinine ratio, and the presence of macrovascular- and microvascular complications.

Macrovascular complications were defined as (a history of) angina pectoris, myocardial infarction, percutaneous transluminal coronary angioplasty, coronary artery bypass grafting, cerebrovascular accident or transient ischaemic attack. Microvascular complications were defined as diabetic retinopathy, albuminuria (both micro- and macroalbuminuria) and diabetic peripheral neuropathy. Microalbuminuria was defined as $20-200 \mathrm{mg} / \mathrm{L}$ albumin or an albumin:creatinine ratio between $2.5-25 \mathrm{mg} / \mathrm{mmol}$ in men and $3.5-35 \mathrm{mg} / \mathrm{mmol}$ in women. Macro-albuminuria was defined as $>200 \mathrm{mg} / \mathrm{L}$ albumin or a albumin:creatinine ratio $>25 \mathrm{mg} / \mathrm{mmol}$ and $35 \mathrm{mg} / \mathrm{mmol}$ for men and women, respectively [16]. An ophthalmologist determined the presence of diabetic retinopathy biannually. Foot sensibility was tested with 5.07 Semmes-Weinstein monofilaments. Neuropathy was defined as two or more errors in a test of three, affecting at least one foot. Blood glucose-lowering therapy was categorised into: dietary measures only, oral blood glucose lowering drugs (OBGLDs) including metformin, sulfonylurea derivatives, thiazolidinediones and DDP4 inhibitors, and insulin therapy. HbA1c is expressed in both IFCC-recommended (as $\mathrm{mmol} / \mathrm{mol}$ ) and DCCTderived (as \%) units.

Participants were instructed to be in a fasting state when the blood samples were collected. Venous blood samples were collected into $\mathrm{BD}$ Vacutainer $^{\mathrm{TM}}$ serum tubes, 
centrifuged and the serum was stored directly in aliquots at $-80{ }^{\circ} \mathrm{C}$ until measurement. Serum magnesium, total cholesterol, HDL cholesterol, triglycerides and creatinine (enzymatic method) were measured on a Modular Analytics P-module (Roche Diagnostics, Mannheim, Germany). LDL cholesterol was quantitated indirectly using the Friedewald formula [17]. A magnesium deficiency was defined as serum concentration $<0.7 \mathrm{mmol} / \mathrm{L} \quad[1,13]$. Tosoh G7 standard mode was used to measure HbAlc.

Normally distributed data are presented as mean \pm SD and non-normally distributed data are presented as median [interquartile range]. Normality of variables was assessed using frequency distribution histograms and Q-Q plots. Variables with a non-parametric distribution were $\log _{10}$ transformed before analysis. Univariable analysis for correlation, using the Pearson correlation coefficient, was performed to investigate the association between serum magnesium and other variables. Variables with a $p$ value $<0.1$, not corrected for multiple testing, were checked for confounding by performing partial regression analyses. Next, multivariable linear regression analysis was performed to investigate associations between serum magnesium dependent variable and multiple independent covariates. In this stepwise backward regression model, variables with $p>0.1$ were eliminated. Covariates were used in the multivariable model based on previous literature (diabetes duration, HbAlc, creatinine, use of metformin, insulin, beta-blockers, diuretics $[2,13,18])$ or in case the $p$ value was $\leq 0.1$ in the univariable analysis. The model was checked for collinearity. A two-sided $p$ value $<0.05$ was considered statistically significant. All statistical analyses were performed using SPSS version 24 (IBM, USA).

The study protocol was registered prior to the start of the study (study ID METC 11.10117) and approved by the Medical Ethical Committee of Isala (Zwolle, the Netherlands). The protocol was also registered on clinicaltrials.gov (study ID NCT01570140). All participants gave written informed consent.

\section{Results}

Baseline characteristics of the 929 persons with T2DM are presented in Table 1. The cohort had a mean age of 65 $( \pm 10)$ years, $55 \%$ were male, diabetes duration was 6.5 [3.0-10.1] years, and $\mathrm{HbA} 1 \mathrm{c}$ concentration was $6.7 \pm 0.7 \%$ $(50 \pm 9 \mathrm{mmol} / \mathrm{mol})$. At baseline, $31 \%$ of the participants had a microvascular complication and $25 \%$ had a macrovascular complication. Concerning treatment, $20 \%$ of the participants were treated with diet alone, while $78 \%$ and $13 \%$ of the participants were treated with OBGLD and/or insulin, respectively.
The mean serum magnesium concentration in the cohort was $0.79 \pm 0.08 \mathrm{mmol} / \mathrm{L}$. There were no significant differences in magnesium concentrations between men and women (men: $0.79 \pm 0.07 \mathrm{mmol} / \mathrm{L}$ : women: $0.79 \pm$ $0.08 \mathrm{mmol} / \mathrm{L}, p=0.17)$. Persons with HbA1c concentrations $<7.0 \%(<53 \mathrm{mmol} / \mathrm{mol})(n=629)$ had lower magnesium concentrations as compared to persons with higher HbAlc levels $(0.80 \pm 0.08 \mathrm{mmol} / \mathrm{L}$ vs. $0.77 \pm 0.07 \mathrm{mmol} / \mathrm{L}$, $p<0.05)$. Hypomagnesaemia was present in $89(9.6 \%)$ persons. In univariable regression analysis, a negative correlation between serum total magnesium concentrations and age, diabetes duration, BMI, HbAlc, use of metformin, sulfonylurea derivatives, DPP4 inhibitors, insulin and RAAS blocking medication was demonstrated (see Table 1). In contrast there was a positive correlation between magnesium and total cholesterol, total cholesterol:HDL ratio, LDL cholesterol, serum creatinine. There was no correlation of serum magnesium with triglycerides or the presence or absence of complications.

In multivariable regression analyses, age, diabetes duration, BMI, HbAlc, the use of metformin, sulfonylurea derivatives and DPP4 inhibitors were negatively associated with serum magnesium concentrations (see Table 2). On the other hand, LDL cholesterol and creatinine were positively associated with magnesium concentrations.

\section{Discussion}

In this cohort of 929 persons with T2DM treated in primary care hypomagnesaemia was present in $9.6 \%$ of all participants. Increasing age, diabetes duration, BMI, HbAlc and the use of metformin, sulfonylurea derivatives and DPP4 inhibitors were associated with low magnesium concentrations while LDL cholesterol and creatinine were associated with higher concentrations.

Interestingly, the prevalence of hypomagnesaemia found in the present study is considerably lower as compared to previous studies, which reported prevalences varying from 14 to $48 \%$ [2]. It is unlikely that the assay system used to determine serum magnesium could account for these differences. In all studies a colorimetric assay was used. Differences in the population studied may account for the differences found. There were barely exclusion criteria for the present study; therefore our results reflect real-life clinical practice. Differences in $\mathrm{HbA} 1 \mathrm{c}$ levels could also explain differences, as magnesium is correlated with blood glucose $[13,19,20]$. Unfortunately, not all previous studies reported $\mathrm{HbAlc}$ levels. In the study by Kurstjens $30.6 \%$ of the patients had a hypomagnesaemia and overall mean HbA1c was $7.9 \pm 1.28 \%(63 \pm 14 \mathrm{mmol} / \mathrm{L})$ : both are higher as compared with the current study. 
Table 1 Baseline characteristics and univariable analysis of correlation with magnesium

\begin{tabular}{|c|c|c|c|}
\hline & Overall $N=931$ & $\begin{array}{l}\text { Univariable Pearson correlation } \\
\text { coefficient }\end{array}$ & $P$-value \\
\hline \multicolumn{4}{|l|}{ Demographics } \\
\hline Age, years & $65.1 \pm 10.1$ & -0.092 & 0.005 \\
\hline Diabetes duration, years & $6.5[3.0-10.1]$ & -0.207 & $<0.001$ \\
\hline Male gender, $n(\%)$ & $508(54.7)$ & 0.045 & 0.169 \\
\hline Current smoker, $n(\%)$ & $154(16.4)$ & 0.035 & 0.289 \\
\hline BMI, $\mathrm{kg} / \mathrm{m}^{2}$ & $29.3[26.8-33.0]$ & -0.140 & $<0.001$ \\
\hline Systolic blood pressure, $\mathrm{mmHg}$ & $135.6[125.0-144.0]$ & -0.064 & 0.054 \\
\hline Diastolic blood pressure, $\mathrm{mmHg}$ & $80.0[71.0-83.0]$ & 0.009 & 0.777 \\
\hline \multicolumn{4}{|l|}{ Complications } \\
\hline \multicolumn{4}{|l|}{ Microvascular complications, $n(\%)$} \\
\hline Retinopathy, $n(\%)$ & $38(4.1)$ & -0.008 & 0.801 \\
\hline Neuropathy, $n(\%)$ & $171(18.4)$ & -0.028 & 0.401 \\
\hline Microalbuminuria, $n(\%)$ & $116(12.5)$ & -0.049 & 0.133 \\
\hline Macroalbuminuria, $n(\%)$ & $11(1.2)$ & -0.012 & 0.712 \\
\hline \multicolumn{4}{|l|}{ Macrovascular events, $n(\%)$} \\
\hline Angina pectoris, $n(\%)$ & $71(7.8)$ & -0.036 & 0.272 \\
\hline Myocardial infarction, $n(\%)$ & $79(8.5)$ & -0.043 & 0.190 \\
\hline PTCA, $n(\%)$ & $25(2.7)$ & -0.021 & 0.525 \\
\hline $\mathrm{CABG}, n(\%)$ & $45(4.8)$ & -0.033 & 0.315 \\
\hline TIA, $n(\%)$ & $31(3.3)$ & -0.016 & 0.621 \\
\hline CVA, $n(\%)$ & $55(5.9)$ & 0.002 & 0.941 \\
\hline Heart failure, $n(\%)$ & $24(2.6)$ & -0.023 & 0.485 \\
\hline \multicolumn{4}{|l|}{ Laboratory measures } \\
\hline $\mathrm{HbAlc}, \mathrm{mmol} / \mathrm{mol}(\%)$ & $6.7 \pm 0.7(50 \pm 9)$ & -0.181 & $<0.001$ \\
\hline eGFR (MDRD), $\mathrm{mL} / \mathrm{min} / 1.73 \mathrm{~m}^{2}$ & $73.0[61.0-86.0]$ & -0.055 & 0.094 \\
\hline Total cholesterol, $\mathrm{mmol} / \mathrm{L}$ & $4.4[3.7-5.0]$ & 0.142 & $<0.001$ \\
\hline HDL cholesterol, mmol/L & $1.3[1.0-1.5]$ & -0.003 & 0.923 \\
\hline Total cholesterol:HDL ratio & $3.4[2.8-4.2]$ & 0.114 & $<0.001$ \\
\hline LDL cholesterol, $\mathrm{mmol} / \mathrm{L}$ & $2.3[1.8-2.8]$ & 0.166 & $<0.001$ \\
\hline Triglycerides, $\mathrm{mmol} / \mathrm{L}$ & $1.5[1.1-2.1]$ & 0.002 & 0.941 \\
\hline Creatinine, $\mu \mathrm{mol} / \mathrm{L}$ & $78.0[67.5-90.0]$ & 0.104 & 0.002 \\
\hline Urine albumine:creatinine ratio, $\mathrm{mg} / \mathrm{mmol}$ & $0.8[0.4-1.5]$ & -0.076 & 0.027 \\
\hline \multicolumn{4}{|l|}{ Treatment } \\
\hline Dietary measures only, $n(\%)$ & $181(19.5)$ & 0.132 & $<0.001$ \\
\hline Oral blood glucose lowering drugs, $n(\%)$ & $725(78.0)$ & & \\
\hline Metformin, $n(\%)$ & $691(74.4)$ & -0.110 & 0.001 \\
\hline Sulfonylurea derivatives, $n(\%)$ & $260(28.0)$ & -0.132 & $<0.001$ \\
\hline Thiazolinediones, $n(\%)$ & $10(1.1)$ & 0.021 & 0.515 \\
\hline DPP4 inhibitors, $n(\%)$ & $35(3.8)$ & -0.069 & 0.035 \\
\hline Insulin, $n(\%)$ & $120(12.9)$ & -0.100 & 0.002 \\
\hline Antihypertensive drugs, $n(\%)$ & $784(84.4)$ & & \\
\hline Diuretics, $n(\%)$ & $348(37.5)$ & -0.029 & 0.389 \\
\hline Beta-blockers, $n(\%)$ & $350(37.5)$ & -0.041 & 0.226 \\
\hline Calcium antagonists, $n(\%)$ & $169(18.2)$ & -0.014 & 0.676 \\
\hline RAAS blockers, n (\%) & $507(54.6)$ & -0.091 & 0.007 \\
\hline Cholesterol lowering drugs, $n(\%)$ & $738(79.4)$ & & \\
\hline Statins, $n(\%)$ & $679(73.1)$ & -0.061 & 0.076 \\
\hline Fibrates, $n(\%)$ & $6(0.6)$ & 0.043 & 0.213 \\
\hline Thrombocyte aggregation inhibitors, $n(\%)$ & $166(17.9)$ & -0.064 & 0.052 \\
\hline
\end{tabular}

Data are presented as number (\%), mean (SD) or median [IQR]. Variables with a non-parametric distribution were $\log _{10}$ transformed before calculation of the Pearson correlation coefficient. Significant $(p<0.05)$ values are highlighted in bold. HbA1c is expressed in both IFCC derived $\mathrm{mmol} / \mathrm{mol}$ as DCCT derived \%

$B M I$ body mass index, $M I$ myocardial infarction, $P T C A$ percutaneous transluminal coronary angioplasty, $C A B G$ coronary artery bypass grafting, TIA transient ischaemic attack, CVA cerebral vascular event, $H b A 1 c$ glycated haemoglobin, $e G F R$ estimated glomerular filtration rate, $H D L$ highdensity lipoprotein, $L D L$ high-density lipoprotein, $D D P 4$ dipeptidyl peptidase $4, R A A S$ renin-angiotensin-aldosterone system 
Table 2 Stepwise multivariable regression analysis with baseline total magnesium concentrations as dependent variable

\begin{tabular}{|c|c|c|c|}
\hline & St. Beta & $P$-value & $\begin{array}{l}\text { Partial } \\
\text { correlation }\end{array}$ \\
\hline Age, years & -0.111 & 0.002 & -0.101 \\
\hline $\log _{10}$ Diabetes duration, years & -0.120 & 0.001 & -0.110 \\
\hline $\log _{10}$ BMI, $\mathrm{kg} / \mathrm{m}^{2}$ & -0.156 & 0.001 & -0.149 \\
\hline $\mathrm{HbA} 1 \mathrm{c}, \%$ & -0.101 & 0.003 & -0.096 \\
\hline $\log _{10}$ LDL cholesterol, $\mathrm{mmol} / \mathrm{L}$ & 0.141 & 0.001 & 0.138 \\
\hline $\log _{10}$ creatinine & 0.109 & 0.001 & 0.105 \\
\hline Metformin* & -0.077 & 0.019 & -0.075 \\
\hline Sulfonylurea derivatives* & -0.085 & 0.010 & -0.082 \\
\hline DPP4 inhibitors* & -0.064 & 0.047 & -0.063 \\
\hline RAAS blockers* & -0.061 & 0.057 & -0.061 \\
\hline Thrombocyte aggregation inhibitors & -0.062 & 0.054 & -0.061 \\
\hline
\end{tabular}

$*$ No $=0$, yes $=1$. Significant values are expressed in bold. Adjusted $R^{2}$ is 0.128 for the model

Another explanation could be the severity of the T2DM. Most previous studies, that found higher prevalence of hypomagnesaemia as compared with our study, were performed in secondary or even tertiary care. In these studies nearly most patient were poorly regulated, had a mean diabetes duration of $>10$ years, were treated with blood glucose lowering medication and frequently also used a significant amount of magnesium lowering co-medication. In the current primary care treated population, $19.5 \%$ of patients used only dietary measures as T2DM treatment, indicating acceptable glycaemic control, and had a median diabetes duration of 6.5 years. Although our findings may indicate that the magnitude of hypomagnesaemia in T2DM is overestimated by previous clinical studies. Nevertheless, hypomagnesaemia still remain a reason for concern, in particular in the light of data linking low magnesium concentrations to arterial calcification and subsequent cardiometabolic comorbidity [21, 22]. Remarkably, we did not find an association between serum magnesium concentrations and the presence of macrovascular complications.

In accordance with previous studies we found a negative association between magnesium concentrations and $\mathrm{HbAlc}$. This may be explained by decreased tubular reabsorption caused by hyperglycaemia and/or hyperfiltration $[10,18,20]$. Of further relevance we did not observe a significant association between magnesium and triglycerides. As a post-hoc univariable analysis among participants not using cholesterol-lowering therapy $(n=190)$, we also observed no association between serum magnesium and triglycerides (St. beta 0.033 , adjusted $R^{2}-0.004, p=$ 0.651 ). This is in contrast to other studies that did find an association among persons with T2DM (20) also independent of waist circumference and BMI [13]. Although the low number of persons may have accounted for the lack of association in the current post-hoc analysis it may also indicate that there is no relevant correlation between magnesium and triglycerides in an unselected Type 2 diabetic population. Likewise, we also found no association of serum magnesium with triglycerides in the PREVEND cohort $[18,23]$. The PREVEND cohort consists of persons from the general population living in the Northern part of the Netherlands. Of notice, the prevalence of a hypomagnesaemia $(<0.7 \mathrm{mmol} / \mathrm{L}$, measured using Roche Modular colourimetric assay) was $1.3 \%$ (72 out of 5568 persons) amongst all persons and 6.6\% (18 out of 272 persons) amongst the persons with diabetes (data derived from $[3,18])$.

In this cohort the use of DPP4 inhibitors seem to lower magnesium concentrations. To the best of our knowledge, this has not been described before. Based on the working mechanism of DPP4 inhibitors and previous literature, we cannot explain this unexpected finding. On the contrary; it was demonstrated recently that the glucagon-like peptide-1 receptor agonist lixisenatide, which also intervenes on the incretin system, reduces renal magnesium excretion in T2DM subjects. The use of DPP4 inhibitors is a third line treatment option in the Netherlands (after dietary measures, metformin and a sulfonylurea derivative) for patient with often a BMI between 30 and $35 \mathrm{~kg} / \mathrm{m}^{2}$. Along the same line with previously discussed, influences of the stage of diabetes this could also be of influence here. However, no multicollinearity was present in our statistical multivariable model, i.e. there was no interaction between BMI, diabetes duration and use of DPP4 inhibitors. Taken together, the relation of DPP4 inhibitors and magnesium needs confirmation and further exploration in future studies.

Loop and thiazide diuretics are well known to lower magnesium concentrations. It is therefore quite remarkable that there was no association between the use of diuretics and magnesium in our cohort. This might be explained by the fact that also potassium-sparing diuretics, that lower magnesium excretion, were included in the same group of diuretics. Also the limited number of patients could be involved here.

It should be emphasised that serum magnesium concentrations were used as a surrogate for total magnesium concentration. This has pros and cons. Magnesium is the second most prevalent intracellular cation; this fraction accounts for $\sim 45 \%$ of the total body magnesium. Extracellular magnesium accounts for $1 \%$ of the total body magnesium content, with the remainder in the skeleton. Approximately $55 \%$ of extracellular magnesium is free, $30 \%$ is associated with proteins, and primarily albumin and $15 \%$ is complexed with anions. Furthermore, it should be emphasised that non-DM related causes of 
hypomagnesaemia such as decreased intake or malabsorption, use of over the-counter-medication including proton pump inhibitors, increased gastro-intestinal loss e.g. due to diarrhoea or medication cannot be excluded in the present cohort. Furthermore, although most currently used blood glucose lowering medications were analysed in this study, data on sodium-glucose co-transporter-2 (SGLT) inhibitors lack, because at the time of study this class of medication was not yet available for clinical uses in the Netherlands. This may become a concern in future as SGLT2 inhibitors may increase urinary magnesium excretion [24].

Other limitations of the present study should be mentioned as well. Due to the cross-sectional nature of the study, we are unable to prove causality between magnesium concentrations and disease status. This also precludes predictions on the development of the comorbidities. For $45 \%$ of potentially eligible participants with T2DM we were unable to match blood samples with clinical data. In addition our cohort is from a single geographic region and consists predominantly (98.8\%) of Caucasians, limiting generalisation of our data. On the other hand, strengths of the study include the large cohort size and the extensive clinical and biochemical characterisation of our participants.

Taken together, in this well-defined cohort of 929 outpatients with T2DM treated in primary care, increasing age, diabetes duration, BMI, HbA1c, the use of metformin, sulfonylurea derivatives, DPP4 inhibitors and RAAS blockers were significantly associated with lower serum magnesium concentrations, while LDL cholesterol and creatinine were associated with higher concentrations. Overall, hypomagnesaemia was present in $9.6 \%$ of all participants. This percentage is remarkably lower than found in previous studies and may suggest that the prevalence of hypomagnesaemia among persons with T2DM treated in primary care is lower than previously suggested.

Acknowledgements The authors want to thank Marian Bulthuis (University Medical Centre, Department of Pathology and Medical Biology, Groningen, the Netherlands) and the Department of Clinical Chemistry of Isala (Zwolle, The Netherlands). This work was supported by grants from the Isala Innovatie and Wetenschapsfonds (grant number INNO1717) and the Zwols Wetenschapsfonds Isala Klinieken. Both the Isala Innovatie and Wetenschapsfonds and Zwols Wetenschapsfonds Isala Klinieken had no role in the study design, data collection, analysis, interpretation, or in the writing of the report.

\section{Compliance with ethical standards}

Conflict of interest The authors declare that they have no conflict of interest.

Ethical approval The study protocol was registered prior to the start of the study (study ID METC 11.10117) and approved by the Medical Ethical Committee of Isala (Zwolle, the Netherlands). The protocol was also registered on clinicaltrials.gov (study ID NCT01570140).
Informed consent Written and verbal informed consent was obtained from all subjects involved in this study.

Publisher's note Springer Nature remains neutral with regard to jurisdictional claims in published maps and institutional affiliations.

Open Access This article is distributed under the terms of the Creative Commons Attribution 4.0 International License (http://crea tivecommons.org/licenses/by/4.0/), which permits unrestricted use, distribution, and reproduction in any medium, provided you give appropriate credit to the original author(s) and the source, provide a link to the Creative Commons license, and indicate if changes were made.

\section{References}

1. L.M.M. Gommers, J.G.J. Hoenderop, R.J.M. Bindels, J.H.F. de Baaij, Hypomagnesemia in type 2 diabetes: a vicious circle? Diabetes 65, 3-13 (2016). https://doi.org/10.2337/db15-1028

2. P.-C.T. Pham, P.-M.T. Pham, S.V. Pham et al. Hypomagnesemia in patients with type 2 diabetes. Clin. J. Am. Soc. Nephrol. 2, 366-373 (2007). https://doi.org/10.2215/CJN.02960906

3. J.C. Schutten, A.W. Gomes-Neto., G. Navis, et al. Lower plasma magnesium, measured by nuclear magnetic resonance spectroscopy, is associated with increased risk of developing type 2 diabetes mellitus in women: results from a Dutch prospective cohort study. J. Clin. Med. 8, 1-16 (2019). https://doi.org/10. 3390/jcm8020169

4. L.E. Simental-Mendía, A. Sahebkar, M. Rodríguez-Morán, F. Guerrero-Romero, A systematic review and meta-analysis of randomized controlled trials on the effects of magnesium supplementation on insulin sensitivity and glucose control. Pharmacol. Res. 111, 272-282 (2016). https://doi.org/10.1016/j.phrs. 2016.06.019

5. J.L. Nadler, T. Buchanan, R. Natarajan et al. Magnesium deficiency produces insulin resistance and increased thromboxane synthesis. Hypertens. Dallas Tex. 1979 21, 1024-1029 (1993)

6. G. Paolisso, S. Sgambato, A. Gambardella et al. Daily magnesium supplements improve glucose handling in elderly subjects. Am. J. Clin. Nutr. 55, 1161-1167 (1992). https://doi.org/10.1093/ajcn/ 55.6.1161

7. A. Hruby, J.B. Meigs, C.J. O’Donnell et al. Higher magnesium intake reduces risk of impaired glucose and insulin metabolism and progression from prediabetes to diabetes in middle-aged americans. Diabetes Care 37, 419-427 (2014). https://doi.org/10. 2337/dc13-1397

8. J. Ma, A.R. Folsom, S.L. Melnick et al. Associations of serum and dietary magnesium with cardiovascular disease, hypertension, diabetes, insulin, and carotid arterial wall thickness: the ARIC study. Atherosclerosis Risk in Communities Study. J. Clin. Epidemiol. 48, 927-940 (1995)

9. M. de Lordes Lima, T. Cruz, J.C. Pousada et al. The effect of magnesium supplementation in increasing doses on the control of type 2 diabetes. Diabetes Care 21, 682-686 (1998)

10. P. McNair, M.S. Christensen, C. Christiansen et al. Renal hypomagnesaemia in human diabetes mellitus: its relation to glucose homeostasis. Eur. J. Clin. Investig. 12, 81-85 (1982)

11. M.K. Wälti, M.B. Zimmermann, G.A. Spinas, R.F. Hurrell, Low plasma magnesium in type 2 diabetes. Swiss Med. Wkly 133, 289-292 (2003). https://doi.org/10.2003/19/smw-10170.

12. N. Mikhail, K. Ehsanipoor, Ionized serum magnesium in type 2 diabetes mellitus: its correlation with total serum magnesium and hemoglobin A1c levels. South Med. J. 92, 1162-1166 (1999) 
13. S. Kurstjens, J.H.F. de Baaij, H. Bouras et al. Determinants of hypomagnesemia in patients with type 2 diabetes mellitus. Eur. J. Endocrinol. 176, 11-19 (2017). https://doi.org/10.1530/EJE16-0517

14. Y. Roelofsen, S.H. Hendriks, F. Sieverink et al. Design of the eVita diabetes mellitus study: effects and use of an interactive online care platform in patients with type 2 diabetes (e-VitaDM-1/ ZODIAC-40). BMC Endocr. Disord. 14, 22 (2014). https://doi. org/10.1186/1472-6823-14-22

15. S.H. Hendriks, E.G.W. van Soldt, M. van Vugt et al. Lifestyle and emotional well-being in men and women with type 2 diabetes (eVitaDM-4; ZODIAC-48). Eur. J. Gen. Pract. 23, 83-90 (2017). https://doi.org/10.1080/13814788.2017.1292348

16. International Diabetes Federation Guideline Development Group, Global guideline for type 2 diabetes. Diabetes Res. Clin. Pract. 104, 1-52 (2014). https://doi.org/10.1016/j.diabres.2012. 10.001

17. W.T. Friedewald, R.I. Levy, D.S. Fredrickson, Estimation of the concentration of low-density lipoprotein cholesterol in plasma, without use of the preparative ultracentrifuge. Clin. Chem. 18, 499-502 (1972)

18. P.R. van Dijk, J.C. Schutten, E.J. Jeyarajah, et al. Blood $\mathrm{Mg}^{2+}$ is more closely associated with hyperglycaemia than with hyper- triacylglycerolaemia: the PREVEND study. Diabetologia (2019). https://doi.org/10.1007/s00125-019-4929-7

19. H.M. Mather, J.A. Nisbet, G.H. Burton et al. Hypomagnesaemia in diabetes. Clin. Chim. Acta Int. J. Clin. Chem. 95, 235-242 (1979). https://doi.org/10.1016/0009-8981(79)90364-4

20. H. Saeed, S. Haj, B. Qasim, Estimation of magnesium level in type 2 diabetes mellitus and its correlation with $\mathrm{HbA} 1 \mathrm{c}$ level. Endocrinol. Diabetes Metab. 2, e00048 (2019). https://doi.org/10. $1002 /$ edm 2.48

21. M. Barbagallo, L.J. Dominguez, Magnesium and type 2 diabetes. World J. Diabetes 6, 1152-1157 (2015). https://doi.org/10.4239/w jd.v6.i10.1152

22. A.D. Ter Braake, C.M. Shanahan, J.H.F. de Baaij, Magnesium counteracts vascular calcification: passive interference or active modulation? Arterioscler Thromb. Vasc. Biol. 37, 1431-1445 (2017). https://doi.org/10.1161/ATVBAHA.117.309182

23. C. Schnack, I. Bauer, P. Pregant et al. Hypomagnesaemia in type 2 (non-insulin-dependent) diabetes mellitus is not corrected by improvement of long-term metabolic control. Diabetologia 35, 77-79 (1992)

24. J.P. Ly, T. Onay, K. Sison et al. The Sweet Pee model for Sglt2 mutation. J. Am. Soc. Nephrol 22, 113-123 (2011). https://doi. org/10.1681/ASN.2010080888 